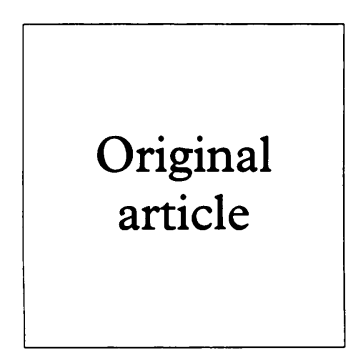

\title{
Utilisation of genitourinary medicine services by general practitioners: effect of geographic location, fundholding status, and potential effect of total fundholding
}

\author{
P D Woolley, S Chandiok
}

Objectives: To determine the current utilisation of genitourinary medicine (GUM) services by general practitioners (GPs) and any changes likely to occur with moves towards a primary care led NHS.

Methods: A questionnaire survey of practising GPs appearing in the Medical Directory in England and Wales to determine the effect of geographical location, fundholding status, and potential effect of total fundholding on referrals to GUM clinics.

Results: Referral patterns for STDs varied according to the genital infection concerned, being highest for gonorrhoea and lowest for Trichomonas vaginalis infection. Referral patterns were little affected by geographic location of the practice or fundholding status. The reported intention on becoming total fundholding was a modest shift towards more STDs being treated in the community which varied according to the genital infection concerned.

Conclusions: Although some GPs elect to always treat some STDs in the community most refer patients to GUM clinics. There appears to be little enthusiasm among GPs, should they become total fundholders, to extend their range of services to include STD provision.

(Genitourin Med 1997;73:538-540)

Keywords: general practitioners; genitourinary medicine clinics; fundholding

\section{Introduction}

In recognition of the public health needs of the population with regard to sexually transmitted diseases (STDs), free and confidential treatment became available decades before the formation of the National Health Service (NHS). Such institutions for the treatment of STDs have since evolved into the present specialty of genitourinary medicine (GUM) which deals with an increasing number of sexual health issues. Returns must be submitted each quarter to the Department of Health indicating the diagnosis of all new cases seen at GUM clinics in England. ${ }^{1}$ It is, however, becoming increasingly apparent that some patient episodes are treated in the community by general practitioners (GPs). ${ }^{23}$

GUM services were made exempt from the initial GP fundholding model of care; funding being provided by a "block" contract. However, the service is not exempt in the total fundholding model and with the shift in emphasis towards a primary care led NHS, it is likely that more GPs will wish to treat their patients with STDs in the community.

The aims of this study were to determine the referral patterns of GPs in England and Wales when presented with patients with STDs and assess whether this is influenced by geographic location or fundholding status by means of a questionnaire survey. Additionally, information was gathered as to reported intention should the GP become a total fundholder.

\section{Methods}

The survey was undertaken in 1995 during which a single page questionnaire was sent to GPs in England and Wales chosen at random from the Medical Directory, until more than 1500 replies had been received; estimated to be a $5 \%$ sample of those practising.

The first part of the questionnaire consisted of demographic data relating to fundholding status and geographic location of the practice self assessed as either rural, mixed (semirural/semiurban), or urban. The second part asked for their usual management from a list of genital infections-possible responses being "always treat" in the community, "sometimes treat", "never treat", or refer to the GUM clinic. The final part of the questionnaire asked GPs to indicate, from the same list of STDs, whether, if they were to become total fundholders and had to purchase STD services for their patients, would they be likely to change their management to "always treat" in the community-possible responses being "yes", "maybe", or "no".

\section{Results}

A total of 1584 questionnaires were returned completed. The response rate was $73 \%$ and gave an estimated sample size of more than $5 \%$ of GPs in England and Wales. As might be expected, only $14 \cdot 7 \%$ of GPs practised exclusively in a rural practice, the rest having some 
Table 1 Current utilisation of GUM services by general practitioners

\begin{tabular}{lcll}
\hline & $\begin{array}{l}\text { Always treat } \\
(\%)\end{array}$ & $\begin{array}{l}\text { Sometimes treat } \\
(\%)\end{array}$ & $\begin{array}{l}\text { Never treat/refer } \\
(\%)\end{array}$ \\
\hline Gonorrhoea & $30(1 \cdot 9)$ & $245(15 \cdot 5)$ & $1309(82 \cdot 6)$ \\
Chlamydia & $241(15 \cdot 2)$ & $947(59 \cdot 8)$ & $396(25)$ \\
NGU & $118(7 \cdot 4)$ & $776(49)$ & $690(43 \cdot 6)$ \\
Genital warts & $95(6)$ & $712(44 \cdot 9)$ & $777(49 \cdot 1)$ \\
Genital herpes & $176(59 \cdot 4)$ & $891(56 \cdot 3)$ & $517(32 \cdot 6)$ \\
Trichomoniasis & $941(59 \cdot 4)$ & $505(31 \cdot 9)$ & $138(8 \cdot 7)$ \\
\hline
\end{tabular}

degree of urban component to their practice. Over half $(56.3 \%)$ of responding GPs were non-fundholding.

\section{CURRENT USE OF GENITOURINARY MEDICINE SERVICES}

The current utilisation of GUM services by GPs when their patients present with gonococcal infection, chlamydial infection, non-gonococcal urethritis (NGU), genital warts, genital herpes, or trichomoniasis is shown in table 1.

Gonococcal infection appears to be the least likely pathogen that GPs are prepared to manage exclusively in the community, with less than $2 \%$ exclusively doing so. $T$ vaginalis infection is the most likely STD to be managed in the community.

\section{GEOGRAPHIC LOCATION}

Despite urban practising GPs presumably having easier geographic access to GUM services than rural practising GPs, the former are more likely to treat gonococcal infection $(2.6 \% v$ $0.9 \%$ ) and, to a much lesser extent, $T$ vaginalis infection $(59.7 \% v 56.5 \%)$. For all other STDs, rural practising GPs are more likely to always treat in the community than urban practising GPs.

\section{FUNDHOLDING STATUS}

At present GUM services are free to both patient and GP (regardless of fundholding status). Although there was a trend towards nonfundholding GPs being more likely to always manage gonorrhoea $(2.1 \% v 1.6 \%)$ and trichomoniasis $(60.7 \% v 57.8 \%)$ in the community there was little difference with respect to the other genital infections.

\section{TOTAL FUNDHOLDING}

Table 2 shows the GPs' reported intention should they become total fundholders. For all infections there was a trend towards managing patients with STDs in the community. There was a fourfold increase in the number of GPs indicating that they would always manage gonorrhoea, and a more than twofold increase with respect to NSU and genital warts, and just under a twofold increase for chlamydial infection and genital herpes.

Table 2 Reported intention of general practitioners if total fundholding on managing STDs in the community

\begin{tabular}{lcrlr}
\hline & $\begin{array}{l}\text { Always } \\
\text { treat }(\%)\end{array}$ & $\begin{array}{l}\text { Maybe } \\
\text { treat }(\%)\end{array}$ & $\begin{array}{l}\text { Sometimes } \\
\text { treat }(\%)\end{array}$ & \multicolumn{1}{c}{$\begin{array}{l}\text { Never treat } \\
\text { Irefer }(\%)\end{array}$} \\
\hline Gonorrhoea & $120(7 \cdot 6)$ & $91(5 \cdot 7)$ & $181(11 \cdot 4)$ & $1192(75 \cdot 3)$ \\
Chlamydia & $395(24 \cdot 9)$ & $104(6 \cdot 6)$ & $742(46 \cdot 8)$ & $343(21 \cdot 7)$ \\
NGU & $267(16 \cdot 8)$ & $107(6 \cdot 8)$ & $614(38 \cdot 8)$ & $596(37 \cdot 6)$ \\
Genital warts & $224(14 \cdot 1)$ & $109(6 \cdot 9)$ & $572(36 \cdot 1)$ & $679(42 \cdot 9)$ \\
Genital herpes & $317(20)$ & $100(6 \cdot 3)$ & $722(45 \cdot 6)$ & $445(28 \cdot 1)$ \\
Trichomoniasis & $1031(65 \cdot 1)$ & $37(2 \cdot 3)$ & $398(25 \cdot 1)$ & $118(7 \cdot 5)$ \\
\hline
\end{tabular}

\section{Discussion}

A response rate of $73 \%$ is respectable for a questionnaire survey of GPs and the sample size of more than $5 \%$ is likely to be representative of GPs in England and Wales. Although only $14.5 \%$ of GPs classified themselves as being in a rural practice, this probably represents the greater degree of urbanisation of GP services and the practical difficulties involved in providing care in a totally rural environment.

It is apparent from this survey that, even with access to a free GUM service, some GPs elect to manage STDs and, in addition, some patients may refuse to attend a clinic. Although GPs seem reluctant to treat gonorrhoea in the community, it is surprising that over $15 \%$ choose to always treat chlamydial infection.

When a person attends a GUM clinic, screening for STDs and treatment play only a part in the total management process. The transmissible nature of genital infections makes them a public health problem and as such partner notification and safer sex counselling vitally important aspects of STD control. It is beyond the scope of this survey to comment on the quality of care afforded to individuals treated for STDs in the community.

GUM services tend to be concentrated in urbanised areas and clinics are commonly attached to the main hospital complex. Consequently, people in rural areas may need to travel long distances to attend a specialist clinic. Whether this influences their decision to attend their GP or not is unknown. However, once they have attended their GP they are more likely to be treated for their STD in the community. The exception to this is gonorrhoea which the rural practising GP appears more willing to refer rather than to treat in the community. This may be due, in part, to the GPs' unwillingness to be involved in the management of this, often emotionally charged, sexually transmitted infection in a close knit community practice.

As GUM services are free to both nonfundholding and fundholding GPs alike, it is not surprising that there is little difference in referral patterns between the two groups. According to GP responses as to their intent should they become total fundholders and therefore be in a position to either provide or contract for STD services, it can be concluded from this survey that some GPs who currently refer patients to a GUM clinic will elect to treat their patients themselves. However, control measures will need to be in place to ensure treatment and management will be to an acceptable standard. Partner notification itself could prove difficult when the sexual partner of a patient treated in the community belongs to a different practice.

Total fundholding GPs electing to treat STDs in the community would be allocated funds presently used to fund local GUM services. In the present financial climate, one obvious consequence of this would be a reduction and contraction of the GUM service 
available. This would make the remaining service less able to respond to any increase in demand should there be any rise in prevalence of STDs.

1 New cases seen at Genitourinary Clinics in England.
Summary information from form KC60. DH Statistical Division 2B.

2 Woolley PD, Chandiok S. Survey of the management of genital herpes in general practice. Int $\mathcal{F}$ STD AIDS 1996; 7:206-11.

3 Estcourt CS, Higgins SP, Hall J, Hillier VF, Chandiok S, Woolley PD. Patients with genital warts: how are they managed by general practioners. Int f STD AIDS 1996; 7:221-3 